\section{(C) OPEN ACCESS}

\title{
Stepwise dose increase of febuxostat is comparable with colchicine prophylaxis for the prevention of gout flares during the initial phase of urate-lowering therapy: results from FORTUNE-1, a prospective, multicentre randomised study
}

\author{
Hisashi Yamanaka, ${ }^{1}$ Shigenori Tamaki, ${ }^{2}$ Yumiko Ide, ${ }^{3}$ Hyeteko Kim, ${ }^{4}$ Kouichi Inoue, \\ Masayuki Sugimoto, ${ }^{6}$ Yuji Hidaka, ${ }^{7}$ Atsuo Taniguchi, ${ }^{1}$ Shin Fujimori, ${ }^{8}$ \\ Tetsuya Yamamoto ${ }^{9}$
}

\begin{abstract}
Handling editor Tore K Kvien
- Additional material is

published online only. To view please visit the journal online (http://dx.doi.org/10.1136/ annrheumdis-2017-211574).

${ }^{1}$ Institute of Rheumatology, Tokyo Women's Medical University, Tokyo, Japan ${ }^{2}$ Nagoya Rheumatology Clinic, Nagoya, Japan

${ }^{3}$ Tokyo Center Clinic, Tokyo, Japan

${ }^{4}$ Yokohama Minoru Clinic, Yokohama, Japan

${ }^{5}$ noue Clinic, Ibaraki, Japan

${ }^{6}$ Shoi-kai Medical Association, Koganeibashi Sakura Clinic, Tokyo, Japan

${ }^{7}$ Taizan-kai Medical Association, Akasaka Central Clinic, Tokyo, Japan

${ }^{8}$ Teikyo University Shinjuku

Clinic, Tokyo, Japan

${ }^{9}$ Department of Diabetes,

Endocrinology and Metabolism, Hyogo College of Medicine, Hyogo, Japan
\end{abstract}

Correspondence to Professor Hisashi Yamanaka, Institute of Rheumatology, Tokyo Women's Medical University 162-0054 10-22 Kawada-cho, Shinjuku-ku,

Tokyo, Japan;

yamanaka@twmu.ac.jp

Received 31 March 2017 Revised 2 October 2017 Accepted 22 October 2017 Published Online First 8 November 2017

\section{CrossMark}

To cite: Yamanaka $\mathrm{H}_{\text {, }}$

Tamaki S, Ide Y, et al.

Ann Rheum Dis

2018;77:270-276

\section{ABSTRACT}

Objectives To determine whether febuxostat with stepwise dose increase is as useful as colchicine prophylaxis in reducing gout flares during the initial introduction of urate-lowering therapy in patients with gout in comparison with febuxostat with no dose titration.

Methods In this prospective, multicentre, randomised open-label comparative study, patients were randomised to group A (stepwise dose increase of febuxostat from 10 to $40 \mathrm{mg} /$ day), group B (fixed-dose febuxostat $40 \mathrm{mg} /$ day plus colchicine $0.5 \mathrm{mg} /$ day) or group C (fixed-dose febuxostat $40 \mathrm{mg} /$ day) and observed for 12 weeks. Gout flare was defined as non-steroidal anti-inflammatory drug use for gout symptoms.

Results A total of 255 patients were randomised, and 241 patients were treated. Among the treated patients, gout flares were experienced by $20 / 96(20.8 \%)$ in group A, $18 / 95(18.9 \%)$ in group B and $18 / 50$ (36.0\%) in group $C$. The incidence of flare was significantly lower in groups $A$ and $B$ than that in group $C(P=0.047$ and $P=0.024$, respectively), although the differences were not significant after correction for multiple comparisons. No significant difference was noted between the incidence of gout flare in groups $A$ and $B$.

Conclusions Our data suggested that stepwise dose increase of febuxostat and low-dose colchicine prophylaxis effectively reduced gout flares in comparison with fixed-dose febuxostat alone. Stepwise dose increase of febuxostat may be an effective alternative to low-dose colchicine prophylaxis during the introduction of uratelowering therapy.

Trial registration number UMIN 000008414.

\section{INTRODUCTION}

The number of patients with gout is increasing, ${ }^{1-3}$ and the debilitating pain of gout flare can severely impact quality of life. In addition, gout and hyperuricaemia are closely associated with diseases related to metabolic syndrome and renal impairment and may be causally related to cardiovascular disease. ${ }^{4-6}$ Gouty arthritis and gouty tophus, clinical presentations of monosodium urate (MSU) crystal deposition, result from persistent hyperuricaemia and can be treated by reducing the body urate pool. This can decrease the long-term incidence of gout flares and urate tophi. ${ }^{7-11}$

However, gout flares frequently develop during the first several months of urate-lowering therapy (ULT). ${ }^{10-12}$ The initial serum urate level, the presence of tophus and the dose of urate-lowering drugs can affect the risk of gout flares during ULT. Unfortunately, medication adherence is poor, ${ }^{13-16}$ partly because gout flares decrease the motivation of patients to continue treatment. ${ }^{1718}$ The prevention of gout flares is thus of key importance when initiating ULT.

Concomitant colchicine can help ${ }^{119}$; recent publications from the European League Against Rheumatism and the American College of Rheumatology recommend colchicine for at least the first 6 months. ${ }^{120}$ However, although widely used for both therapeutic and prophylactic purposes, colchicine is potentially toxic and caution is advised. ${ }^{21-23}$

ULT induces the shedding of deposited MSU crystals in the joints. Such crystal shedding may be facilitated by the dissolution of urate crystals, and also by decreased urate levels in the joint fluid. ${ }^{24}$ Thus, a rapid decrease in serum urate could contribute to gout flares, whereas a gradual decrease should favour flare prevention. ${ }^{10-12}$

In Japan, clinical trials using a stepwise increase in febuxostat dose at the initiation of treatment have shown a lower incidence of gout flares than trials using fixed-dose febuxostat. ${ }^{25} 26$ Thus, there are at least two potential strategies to reduce early treatment-related gout flares: stepwise dose increase and colchicine prophylaxis. However, no prospective clinical trials have been conducted to compare the efficacy of these two strategies.

The present study was designed to investigate the incidence of gout flares during early-stage febuxostat treatment, comparing fixed-dose monotherapy both to stepwise dose increase and to low-dose colchicine prophylaxis. 


\section{PATIENTS AND METHODS}

\section{Patients}

Men with gout who had at least one episode of gouty arthritis within 1 year before study entry, whose serum urate exceeded $7.0 \mathrm{mg} / \mathrm{dL}(416.39 \mu \mathrm{mol} / \mathrm{L})$ and who had not received treatment with any urate-lowering drugs for at least 1 month prior to entry were enrolled after giving written informed consent. Diagnosis of gout was based on the 1977 criteria. ${ }^{27}$ Patients experiencing gouty arthritis within 2 weeks before study entry were excluded. Other exclusion criteria were age $<20$ years, history of allergic reaction to febuxostat, colchicine or non-steroidal anti-inflammatory drugs (NSAIDs), presence of serious comorbidities including serum creatinine level of $2.0 \mathrm{mg} / \mathrm{dL}$ or higher and the judgement of the investigator that the patient was not an appropriate candidate for study participation. Inclusion and exclusion criteria are detailed in online supplementary table 1 .

\section{Study design}

This prospective, multicentre, randomised, open-label comparative study was conducted by the Febuxostat Outcome Research Towards Urate Lowering in the Next Decade (FORTUNE) consortium, organised by multiple clinical sites across Japan, ${ }^{28}$ and was designated the FORTUNE-1 study. Patients were randomised as follows: group A, stepwise dose increase of febuxostat from $10 \mathrm{mg} /$ day (4 weeks), $20 \mathrm{mg} /$ day (4 weeks) and $40 \mathrm{mg} /$ day (until the end of the study); group B, febuxostat $40 \mathrm{mg} /$ day from the start of the study, with concomitant colchicine $0.5 \mathrm{mg} /$ day; or group C, febuxostat $40 \mathrm{mg} /$ day from the start of the study. Patients took oral febuxostat one time per day in the morning, or oral febuxostat and colchicine at the same time one time per day in the morning.

This randomised treatment was conducted during the first 12 weeks (randomised period), after which all patients were treated with febuxostat $40 \mathrm{mg}$ /day for another 12 weeks (observation period).

Because we anticipated that the incidence of gout flares might be higher in group $\mathrm{C}$, the randomisation ratio for groups $\mathrm{A}, \mathrm{B}$ and $\mathrm{C}$ was set at 2:2:1 for ethical reasons.

The allocation sequence was computer generated by a data centre (Mebix, Tokyo), using a random number table. Randomisation was by minimisation, ${ }^{29}$ adjusted by baseline serum urate $(<8.0 \mathrm{mg} / \mathrm{dL}(475.88 \mu \mathrm{mol} / \mathrm{L})$ or $\geq 8.0 \mathrm{mg} / \mathrm{dL}(475.88 \mu \mathrm{mol} / \mathrm{L}))$, age $(<50$ or $\geq 50$ years), previous anti-hyperuricaemic therapy $(<1$ or $\geq 1)$ and previous incidence of gouty flare ( 1 or $\geq 2$ per year). The investigators initiated treatment after being informed of the results of assignment by the data centre.

This study used an open-label design; investigators and patients were aware of their treatment arm. Investigators handled patient assessment and data collection.

The attending investigator evaluated each adverse event (AE) and graded the severity as mild (awareness of sign or symptom but no significant discomfort), moderate (discomfort requiring intervention) or severe (prevents daily routine activity or has a clinically important effect). If a causal relationship with febuxostat could not be ruled out, attending physicians could reduce the dose or discontinue febuxostat. During the study, other urate-lowering drugs and drugs that are known to increase or decrease the serum urate level were prohibited. Attending physicians were requested to prescribe NSAIDs at the start of the study for use in managing gout flares. Patients who did not therapeutically respond to NSAIDs or had multiple flares were given corticosteroids.

\section{Sample size}

The sample size for the study was based on the primary end point. A previous study noted that a lower percentage of patients experienced gout flares in groups A and B than that in group C. ${ }^{30}$ Based on earlier reports in the literature ${ }^{252630}$ we estimated that the incidence of gout flares during a 12 -week treatment period would be $5 \%$ for groups A and B and 25\% for group C in this study. Thus, for ethical reasons, we set the ratio of treatment groups at 2:2:1. To achieve a 5\% two-sided significance level and $89 \%$ power to detect the differences between group A and group $\mathrm{C}$ and between group $\mathrm{B}$ and group $\mathrm{C}$, we calculated that 90 patients were required for group A, 90 patients for group B and 45 patients for group C. We estimated a $10 \%$ dropout rate from the study and, thus, set the sample size to 100, 100 and 50 patients, respectively.

\section{End points}

The primary end point was the incidence rate of gouty arthritis (gouty aura not included) during the 'treatment period' (the first 12 weeks of the study), defined as the percentage of patients who needed analgesic treatment with NSAIDs or adrenal corticosteroid to manage gout symptoms.

Patients were instructed to record symptoms and NSAID use in the specified patient record form. Rubor, swelling and other symptoms were verified by the attending investigator during the patient's next visit. Visits occurred every 4 weeks. Mild attacks that were tolerable without NSAID use were not defined as gout flares. Any use of NSAIDs for reasons other than gouty arthritis was excluded from analysis.

The secondary end points included the number of gout flares per patient during the first 12 weeks (randomised period), the number of gout flares per patient during the second 12 weeks (observation period) and the percentage of patients with serum urate $\leq 6.0 \mathrm{mg} / \mathrm{dL}(356.91 \mu \mathrm{mol} / \mathrm{L})$ in the second 12 weeks (observation period).

An AE was defined as any untoward medical occurrence in a subject given the study drug, without requiring a causal relationship to the treatment. An adverse reaction (AR) was defined as any event for which a causal relationship to febuxostat could not be ruled out.

\section{Statistical analysis}

All statistical analyses were performed on the full analysis set (FAS) as defined in the Results section. Baseline patient demographics were presented as mean and SD for continuous variables and as frequency for categorical variables. In the primary end point analysis, the statistical analysis was corrected for multiple comparisons. A Pearson $\chi^{2}$ test on a $3 \times 2$ table was used to compare group A with group C. The higher of the two $\mathrm{P}$ values was used to test for significance using a threshold of 0.05 . This preserves the family-wise error rate when only three groups are involved. ${ }^{31}$

For secondary end point analyses, corrections for multiple testing were not performed.

The Wilcoxon rank-sum test was used for the number of gout flares, and the Fisher exact test was used for the percentage of patients with serum urate $\leq 6.0 \mathrm{mg} / \mathrm{dL}(356.91 \mu \mathrm{mol} / \mathrm{L})$.

All tests were two-sided. Statistical significance was at $\mathrm{P}<0.05$. Baseline demographics were summarised using SAS V.9.4 (SAS Institute). Other analyses were performed using R V.3.0.3..$^{32}$ 


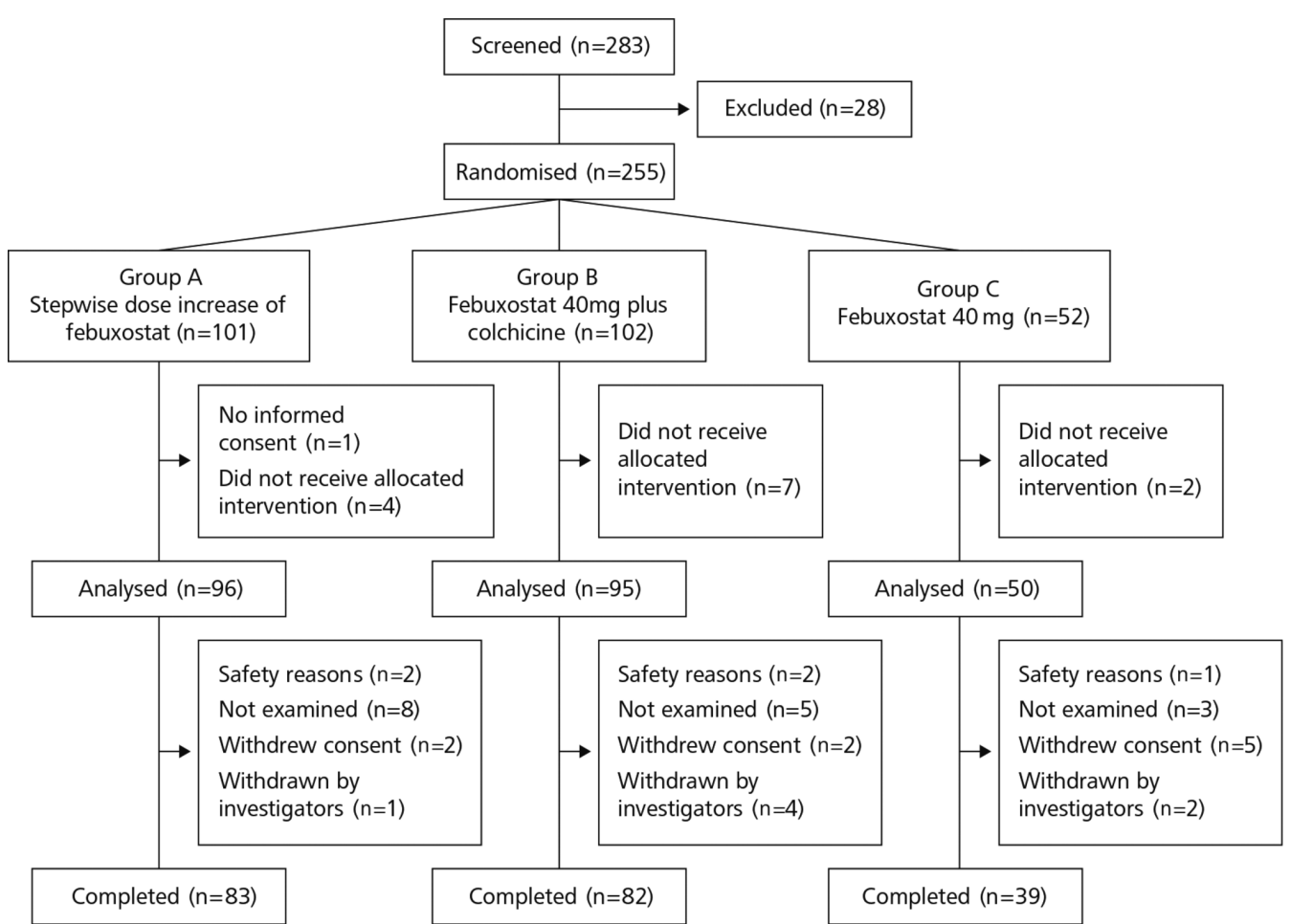

Figure 1 Patient disposition.

\section{RESULTS}

\section{Patient disposition and baseline characteristics}

The trial was conducted at 24 centres between August 2013 and February 2015. As shown in figure 1, a total of 283 patients agreed to participate in this study (intention-to-treat group). After excluding patients who met the exclusion criteria or did not meet the inclusion criteria, we randomised 255 patients (group A, 101; group B, 102; group C, 52) for the study. Patients received treatment for 12 weeks and were monitored for an additional 12 weeks. A total of 14 patients were excluded from primary end point analysis: 13 patients because treatment was declined by the doctor or the patient and 1 patient without informed consent. The remaining 241 patients were defined as the FAS. The data from these 241 patients (group A, 96; group $\mathrm{B}, 95$; group C, 50) were used for subsequent analyses (figure 1).

Table 1 summarises the baseline characteristics of these 241 patients. One-third of the patients had received prior ULT; urate-lowering drugs were washed out for more than 1 month in these patients. No statistically significant differences were noted in the baseline features of the three groups.

\section{Incidence of gout flares in the first 12 weeks (randomised period)}

Gout flares were experienced within the first 12 weeks (randomised period) by 20 of $96(20.8 \%)$ patients in group A, 18 of 95 patients (18.9\%) in group B and 18 of 50 patients $(36.0 \%)$ in group C (figure 2). In an overall Pearson $\chi^{2}$ test, the $\mathrm{P}$ value was 0.054 , and for the comparison of groups $\mathrm{A}$ and $\mathrm{C}$, the $\mathrm{P}$ value was 0.048 . Although this $\mathrm{P}$ value was below 0.05 , the null hypothesis for the primary end point was not rejected because the higher $\mathrm{P}$ value of the two tests $(0.054)$ was above the 0.05 threshold. The difference in flare incidence was statistically significant between group $\mathrm{B}$ and group $\mathrm{C}(\mathrm{P}=0.024)$

Table 1 Baseline demographics of patients (FAS)

\begin{tabular}{|c|c|c|c|}
\hline & Group A $(n=96)$ & Group B $(n=95)$ & Group C $(n=50)$ \\
\hline & Febuxostat dose increasing $10-40 \mathrm{mg}$ & Febuxostat $40 \mathrm{mg}+$ colchicine & Febuxostat $40 \mathrm{mg}$ \\
\hline Age, mean (SD) & $47.4(10.5)$ & $47.6(11.1)$ & $46.4(12.7)$ \\
\hline Height, mean (SD), cm & $171.0(5.7)$ & $170.8(5.8)$ & $169.8(6.9)$ \\
\hline Weight, mean (SD), kg & $77.3(12.4)$ & $76.5(11.7)$ & $76.8(16.4)$ \\
\hline $\mathrm{BMI}$, mean (SD), kg/m² & $26.4(3.6)$ & $26.2(3.5)$ & $26.5(5.1)$ \\
\hline Systolic blood pressure, mean (SD), $\mathrm{mm} \mathrm{Hg}$ & $132.6(14.4)$ & $132.8(16.3)$ & $132.6(17.6)$ \\
\hline Diastolic blood pressure, mean (SD), mm Hg & $84.5(12.0)$ & $84.3(11.9)$ & $82.6(14.0)$ \\
\hline Any history of $\geq 2$ gout flares, $n(\%)$ & $74(77.1)$ & $70(73.7)$ & $38(76.0)$ \\
\hline Prior urate-lowering therapy, $\mathrm{n}(\%)$ & $31(32.3)$ & $31(32.6)$ & $16(32.0)$ \\
\hline eGFR at entry, mean (SD), mL/min $/ 1.73 \mathrm{~m}^{2}$ & $75.8(16.2)$ & $76.6(13.7)$ & $76.8(17.5)$ \\
\hline Serum urate at entry, mean (SD), mg/dL & $8.67(1.38)$ & $8.51(1.19)$ & $8.57(1.17)$ \\
\hline Serum creatinine at entry, mean (SD), mg/dL & $0.90(0.16)$ & $0.88(0.14)$ & $0.89(0.15)$ \\
\hline With any comorbidity, n (\%) & $51(53.1)$ & $47(49.5)$ & $29(58.0)$ \\
\hline
\end{tabular}

BMI, body mass index; eGFR, estimated glomerular filtration rate; FAS, full analysis set. 


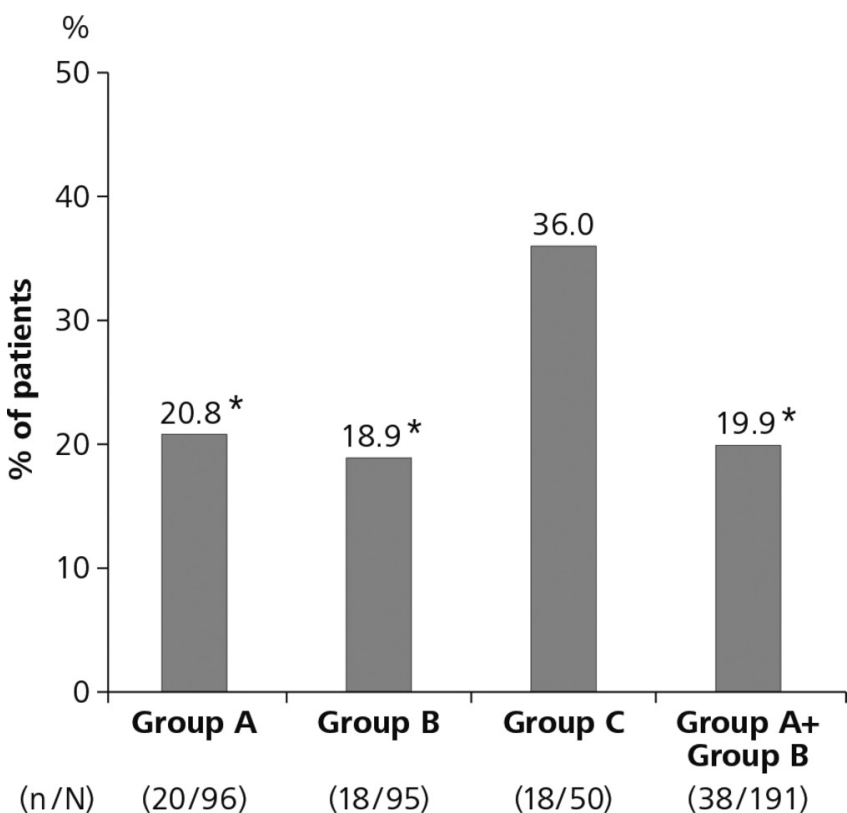

Figure 2 Incidence of gout flares during the randomised period. Incidence of gout flares during the first 12 weeks (randomised period) in group $A$ (stepwise dose increase of febuxostat from 10 to $40 \mathrm{mg} /$ day), group B (febuxostat $40 \mathrm{mg} /$ day plus colchicine $0.5 \mathrm{mg} /$ day) and group C (febuxostat $40 \mathrm{mg} / \mathrm{day}$ ). The overall Pearson $\chi^{2}$ test was not significant. See text for details. ${ }^{*} \mathrm{P}<0.05$ vs group $\mathrm{C}$.

and between group A+group B and group C (19.9\%, 95\% CI 14.2 to $25.6, \mathrm{P}=0.016$ ) but not between group $\mathrm{A}$ and group $\mathrm{B}$ $(\mathrm{P}=0.744)$.

The incidence of gout flare was compared in patients previously treated with ULT and those who were treatment naïve at baseline. There was no significant difference in the incidence of gout flares in patients with or without previous ULT within each treatment group or within the entire study group (online supplementary table 2).

\section{Number of gout flares per patient in the study period}

To investigate the characteristic time course of gout flares in each group, we analysed the number of gout flares in each study period.

During the first 12 weeks (randomised period), a total of 27 flares were identified in 20 patients ( 1.35 flares/patient) in group A, 24 flares in 18 patients (1.33 flares/patient) in group B and 37 flares in 18 patients (2.06 flares/patient) in group C. There was no significant difference between group A and group $\mathrm{C}$ or between group B and group C. In the second 12 weeks (observation period), there were 18 flares in 15 patients (1.20 flares/ patient) in group A, 26 flares in 17 patients (1.53 flares/patient) in group B and 8 flares in six patients (1.33 flares/patient) in group C. There was no significant difference between treatment groups. The number of flares in each patient who had gout flares in the first 12 weeks and the second 12 weeks is illustrated in figure 3A,B, respectively.

During the 24 weeks (randomised + observational periods), there were 45 flares in 30 patients (1.50 flares/patient) in group A, 59 flares in 28 patients (1.79 flares/patient) in group B and 45 flares in 19 patients (2.37 flares/patient) in group C. The number of flares in each patient is illustrated in online supplementary figure 1.
A $\%$
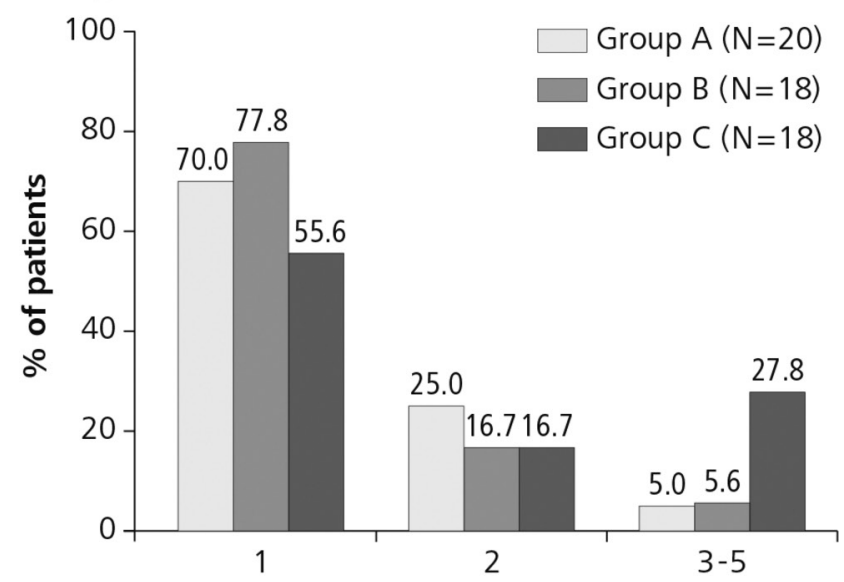
(n)
(14)(14)(10)
(5) (3) (3)
(1) (1) (5)

\section{Number of gout flares}

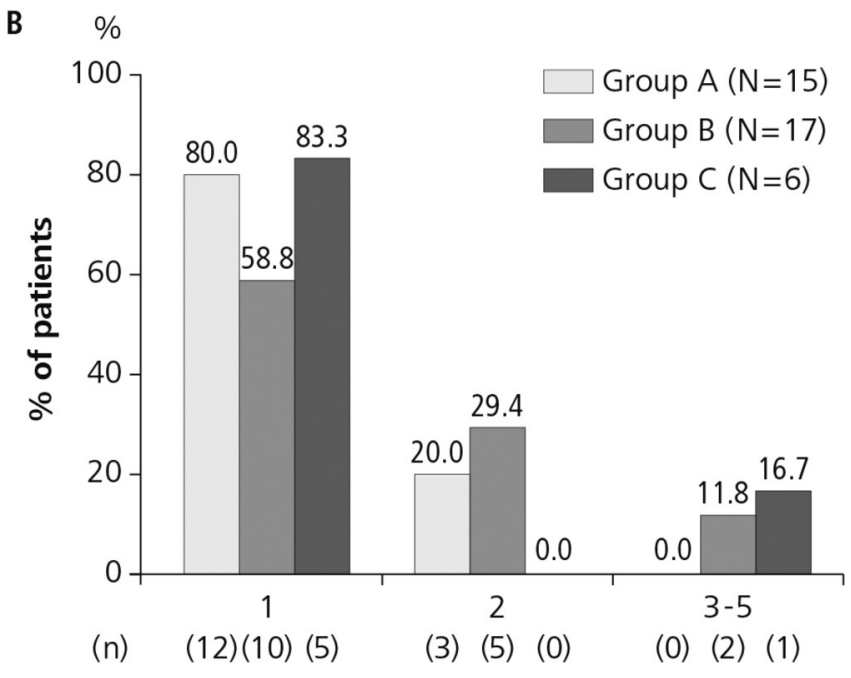

Number of gout flares

Figure 3 Number of gout flares during the study period. Number of gout flares per patient during the first 12 weeks (randomised period) (A) and the second 12 weeks (observation period) (B). Group A (stepwise dose increase of febuxostat from 10 to $40 \mathrm{mg} /$ day), group B (febuxostat $40 \mathrm{mg} /$ day plus colchicine $0.5 \mathrm{mg} /$ day) and group C (febuxostat $40 \mathrm{mg} /$ day).

\section{Percentage of patients with serum urate $\leq 6.0 \mathrm{mg} / \mathrm{dL}$ (356.91 $\mu \mathrm{mol} / \mathrm{L})$}

Urate-lowering effects of treatment were investigated in the 241 patients. Some data were missing because treatment had been discontinued or were otherwise unavailable. Figure 4 shows the percentage of patients whose serum urate decreased to $6.0 \mathrm{mg} /$ dL $(356.91 \mu \mathrm{mol} / \mathrm{L})$ or below at weeks 4, 8, 12, 16, 20 and 24 , and the number of patients used for calculations for each time point and each group.

A significantly lower percentage of patients reached the target level of serum urate at 4 weeks $(P<0.001)$ and 8 weeks $(\mathrm{P}<0.001)$ in group A compared with group B or group $\mathrm{C}$. There was no significant difference among the three treatment groups after 12 weeks.

\section{Safety profile}

AEs and ARs are listed in table 2. No clinically important AEs or serious AEs were reported. No differences were identified in the 


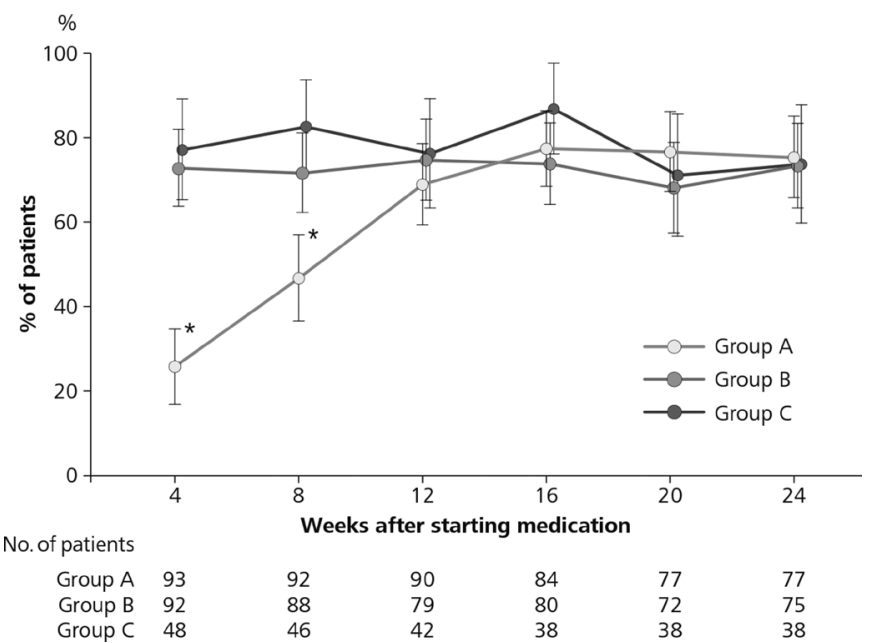

Figure 4 Percentage of patients with serum urate level $\leq 6.0 \mathrm{mg} / \mathrm{dL}$ (356.91 $\mu \mathrm{mol} / \mathrm{L}$ ) in the three randomised groups. Group A (stepwise dose increase of febuxostat from 10 to $40 \mathrm{mg} /$ day), group B (febuxostat $40 \mathrm{mg} /$ day plus colchicine $0.5 \mathrm{mg} / \mathrm{day}$ ) and group C (febuxostat $40 \mathrm{mg} /$ day). The number of patients for calculations based on urate data at each weekly time point in each group is shown below the figure. ${ }^{*} \mathrm{P}<0.001$ vs group $\mathrm{B}$ or group $\mathrm{C}$.

incidence of ARs among the three groups. Moderate diarrhoea was reported in one patient in group B, but not in any patients in group A or group C.

\section{DISCUSSION}

Febuxostat is a xanthine oxidase inhibitor with a potent serum urate-lowering effect and a reliable safety profile. ${ }^{252633}$ In the first clinical studies of febuxostat in Japan, gout flare was the major AE; subsequent study designs incorporated a stepwise dose increase, which reduced the incidence of gout flares. ${ }^{25} 26$ As a result, the official labelling for Feburic (commercial name of febuxostat in Japan) specifies a stepwise increase in the febuxostat dose.

There are two possible ways to reduce gout flares during the initial period of ULT: colchicine prophylaxis and stepwise dose increase. Our study is the first to compare these two strategies, using febuxostat as the urate-lowering drug. As a control, febuxostat $40 \mathrm{mg}$ without colchicine prophylaxis and without stepwise dose increase (group C) was included. For ethical reasons (to minimise the disadvantage to patients), fewer patients were randomised to this arm, and all patients were instructed to take an NSAID if a flare should occur.

The result of the primary end point analysis that included the correction for multiple comparisons was negative. However, as shown in figure 2 , the incidence of gout flares was significantly lower in group A (stepwise dose increase) than that in group $\mathrm{C}$ (without stepwise dose increase or colchicine prophylaxis) and was also significantly lower in group B (low-dose colchicine prophylaxis) than that in group $\mathrm{C}$. These findings were further confirmed by comparing incidence of flares between groups $\mathrm{A}$ plus B and group C.

We found no difference in the incidence or the number of gout flares between group A and group B. This suggests that,

Table 2 Incidence of adverse events and adverse reactions by system organ class (safety population)

\begin{tabular}{|c|c|c|c|c|}
\hline & \multirow[t]{2}{*}{ Total $(n=241)$} & \multirow{2}{*}{$\begin{array}{l}\text { Group A ( } n=96) \\
\text { Febuxostat dose increasing } \\
\text { stepwise from } 10 \text { to } 40 \mathrm{mg}\end{array}$} & \multirow{2}{*}{$\begin{array}{l}\text { Group B }(n=95) \\
\text { Febuxostat } \\
40 \mathrm{mg}+\text { colchicine }\end{array}$} & \multirow{2}{*}{$\begin{array}{l}\text { Group C }(n=50) \\
\text { Febuxostat } 40 \mathrm{mg}\end{array}$} \\
\hline & & & & \\
\hline & Patients (events) \% & Patients (events) \% & Patients (events) \% & Patients (events) \% \\
\hline Adverse events & $51(74) 21.2$ & $21(35) 21.9$ & 19 (23) 20.0 & $11(16) 22.0$ \\
\hline Infections and infestations & $25(35) 10.4$ & $9(15) 9.4$ & $11(13) 11.6$ & $5(7) 10.0$ \\
\hline Neoplasms benign, malignant and unspecified & $1(1) 0.4$ & $1(1) 1.0$ & - & - \\
\hline Metabolism and nutrition disorders & $2(2) 0.8$ & $2(2) 2.1$ & - & - \\
\hline Vascular disorders & $1(1) 0.4$ & $1(1) 1.0$ & - & - \\
\hline Respiratory, thoracic and mediastinal disorders & $4(6) 1.7$ & 2 (4) 2.1 & $1(1) 1.1$ & 1 (1) 2.0 \\
\hline Gastrointestinal disorders & $1(1) 0.4$ & - & $1(1) 1.1$ & - \\
\hline Hepatobiliary disorders & $5(5) 2.1$ & 4 (4) 4.2 & - & $1(1) 2.0$ \\
\hline Skin and subcutaneous tissue disorders & $2(2) 0.8$ & $1(1) 1.0$ & 1 (1) 1.1 & - \\
\hline Musculoskeletal and connective tissue disorders & $9(10) 3.7$ & $4(5) 4.2$ & $3(3) 3.2$ & $2(2) 4.0$ \\
\hline Renal and urinary disorders & $2(2) 0.8$ & $1(1) 1.0$ & $1(1) 1.1$ & - \\
\hline Investigations & $4(4) 1.7$ & - & $3(3) 3.2$ & $1(1) 2.0$ \\
\hline Injury, poisoning and procedural complications & $4(4) 1.7$ & $1(1) 1.0$ & - & $3(3) 6.0$ \\
\hline Surgical and medical procedures & $1(1) 0.4$ & - & - & 1 (1) 2.0 \\
\hline Adverse reactions & $21(24) 8.7$ & 7 (9) 7.3 & $9(10) 9.5$ & $5(5) 10.0$ \\
\hline Infections and infestations & $7(8) 2.9$ & - & $6(7) 6.3$ & $1(1) 2.0$ \\
\hline Metabolism and nutrition disorders & $1(1) 0.4$ & $1(1) 1.0$ & - & - \\
\hline Respiratory, thoracic and mediastinal disorders & $1(1) 0.4$ & - & $1(1) 1.1$ & - \\
\hline Hepatobiliary disorders & $4(4) 1.7$ & 3 (3) 3.1 & - & $1(1) 2.0$ \\
\hline Skin and subcutaneous tissue disorders & $2(2) 0.8$ & $1(1) 1.0$ & $1(1) 1.1$ & - \\
\hline Musculoskeletal and connective tissue disorders & $3(4) 1.2$ & $2(3) 2.1$ & - & $1(1) 2.0$ \\
\hline Renal and urinary disorders & $2(2) 0.8$ & $1(1) 1.0$ & $1(1) 1.1$ & - \\
\hline Investigations & $1(1) 0.4$ & - & - & $1(1) 2.0$ \\
\hline Injury, poisoning and procedural complications & $1(1) 0.4$ & - & - & 1 (1) 2.0 \\
\hline
\end{tabular}


when introducing ULT with febuxostat, stepwise dose increase of febuxostat is better than the single-dose method and is comparable with low-dose colchicine prophylaxis for the prevention of gout flares. Because colchicine prophylaxis has been shown to decrease gout flares during the introduction of allopurinol, ${ }^{34}$ stepwise dose increase of febuxostat is also likely to be beneficial for gout patients.

Non-inferiority or equivalence testing of gout flares between group A and group B would confirm whether febuxostat stepwise dose increase is comparable with colchicine prophylaxis in reducing gout flares. However, such tests were not performed in the present study because of insufficient sample size. Under the current concept of treat to target, the recommended serum urate level is to be maintained below $6.0 \mathrm{mg} / \mathrm{dL}(356.91 \mu \mathrm{mol} / \mathrm{L}){ }^{3536}$ Under stepwise dose increase, lowering of urate to the target level $(6.0 \mathrm{mg} / \mathrm{dL}(356.91 \mu \mathrm{mol} / \mathrm{L}))$ was delayed in group A, but by week 12 , the same percentage of patients had achieved serum urate at or below $6.0 \mathrm{mg} / \mathrm{dL}(356.91 \mu \mathrm{mol} / \mathrm{L})$ as in the other two groups. This suggests that stepwise dose increase is a practical treatment option. This is particularly important because colchicine can be toxic in large amounts, ${ }^{21}{ }^{37}$ suggesting that the use of colchicine should potentially be restricted in patients with multiple comorbidities. Thus, we propose stepwise dose increase of febuxostat as a useful alternative option to minimise the occurrence of gout flares when starting ULT.

Limitations of this study include the open-label design (patients and investigators were informed of the patient's treatment arm, which might have affected study results) and the definition of gout flare (under discussion in the literature ${ }^{38}$; the provisional definition ${ }^{39}$ may not apply in studies of real-world clinical therapy). For this study, we defined gout flares as symptoms of gout requiring NSAID treatment. This definition is not universally accepted, ${ }^{3738}$ but we consider it reasonably useful for comparing the incidence of gout flares among three treatment arms in our study. The severity of gout may also be a consideration. Japanese patients generally have milder forms of gout than patients in the USA or Europe, as measured by the percentage of patients with tophi ${ }^{25} 26$ or the dose of febuxostat required. ${ }^{40}$ The usual dose of febuxostat for lowering serum urate to below $6.0 \mathrm{mg} / \mathrm{dL}(356.91 \mu \mathrm{mol} / \mathrm{L})$ is $40 \mathrm{mg} /$ day in Japan and $80 \mathrm{mg} / \mathrm{day}$ in the USA or Europe, perhaps due to smaller body size and earlier intervention for hyperuricaemia in Japan. Also, the dose of colchicine for gout flare prophylaxis in group B was consistent with Japanese labelling for colchicine but lower than that in other countries. Thus, the results of this study may not be directly applicable to patients with gout in the USA or Europe. Finally, this study may have failed to meet the primary end point because of insufficient sample size, especially in group C. This should influence the design of future studies.

In conclusion, our results suggested that a stepwise dose increase of febuxostat reduced the incidence of gout flares to an extent comparable with low-dose colchicine prophylaxis. Because of issues related to the safety of colchicine, a stepwise dose increase of febuxostat can be a recommended option for reducing the incidence of gout flares. We hope that this strategy can increase patient adherence and improve long-term outcomes.

Correction notice This article has been corrected since it published Online First The corresponding author's email address has been corrected.

Acknowledgements The authors thank the contract research organisation Mebix Corporation (http://www.mebix.co.jp) for data collection and the Nature Insight Corporation (http://www.n-insight.co.jp/) and StaGen (www.stagen.co. jp) for statistical analysis, especially the very helpful explanations from Dr Naoyuki Kamatani and Dr Eisuke Inoue. We also thank Dr Naoyuki Kamatani for his useful comments on the study.
Collaborators The institutions and names of the chief investigators of the FORTUNE-1 study group are listed below: 1. Institute of Rheumatology, Tokyo Women's Medical University, Hisashi Yamanaka (principal investigator); 2. Nagoya Rheumatology Clinic, Shigenori Tamaki; 3. Tokyo Center Clinic, Yumiko Ide; 4. Yokohama Minoru Clinic, Hyeteko Kim; 5. Inoue Clinic, Kouichi Inoue; 6. Syoi-kai Medical Association Koganeibashi Sakura Clinic, Masayuki Sugimoto; 7. Taizankai Medical Association Akasaka Central Clinic, Yuji Hidaka; 8. Taizan-kai Medical Association Toranomon Clinic, Mitsuko Akimoto; 9. Institute of Rheumatology, Tokyo Women's Medical University, Naomi Ichikawa; 10. Sagawa Akira Rheumatology Clinic, Akira Sagawa; 11. Isei-kai Medical Association Sakaibashi Clinic, Hisayoshi Hirota; 12. Jyakuei-kai Medical Association Tanaka Hospital, Tsuneo Tanaka; 13. Kowa-kai Medical Association Jin Orthopedics Clinic, Hiroaki Jin; 14. Koni Clinic, Ichiro Koni; 15. Seiga-kai Medical Association Momoi Orthopedics Clinic, Yasuharu Momoi; 16. Inoue Orthopedics Clinic, Soitiro Inoue; 17. East Sendai Rheumatism and Internal Medicine Clinic, Tomomasa Izumiyama; 18. Seisyu-kai Medical Association Yagi Clinic, Hideyuki Yagi; 19. National Hospital Organization Awara Hospital, Hiroshi Tsutani; 20. Senoo Hospital, Masayuki Hakoda; 21. Tohoku University Hospital, Tomonori Ishii; 22. Nakashima Hiroshi Clinic, Hiroshi Nakashima; 23. Chiba Prefectural Togane Hospital, Aizan Hirai.

Contributors All authors have equal responsibility for the manuscript and meet all the authorship requirements. The corresponding author (HY) organised every stage of the study, including the naming of the study, and was responsible for drafting the manuscript. HY, AT, SF and TY participated in the design of the study and helped to revise the manuscript. All authors were members of the FORTUNE-1 study group, participated in data acquisition and gave suggestions as necessary; those suggestions provided the basis for modifications to the manuscript. All authors have read and approved this revised version of the manuscript for submission. The corresponding author had full responsibility for the study design, supervised the data collection, had full access to all data, supervised data analysis and was responsible for submitting the manuscript for publication.

Funding This investigator-initiated study was supported by a grant from Teijin Pharma Limited. The sponsors were not involved in the design of the study; enrolment of patients; data collection, analysis and interpretation; or preparation of the manuscript.

Competing interests HY reports grants and personal fees from Teijin Pharma, Pfizer Japan, Takeda Pharmaceutical Company, Bristol-Myers Squibb, Nippon Kayaku, Chugai Pharmaceutical, Mitsubishi Tanabe Pharma, Daiichi Sankyo, Astellas Pharma, AbbVie, UCB Japan, Ono Pharmaceutical, Ayumi Pharmaceutical, Eisai, Torii Pharmaceutical, Taisho Toyama Pharmaceutical and YL Biologics, and grants from MSD. ST reports personal fees from Teijin Pharma. YH reports personal fees from Teijin Pharma, Pfizer Japan, Sanwa Kagaku Kenkyusho and Fujiyakuhin. AT reports personal fees from Teijin Pharma. SF reports personal fees from Teijin Pharma, Sanwa Kagaku Kenkyusho, Fujiyakuhin, Astellas Pharma, Kissei Pharmaceutical, Pfizer Japan, MSD and Nippon Chemiphar. TY reports personal fees and non-financial support from Teijin Pharma.

\section{Patient consent Obtained.}

Ethics approval The study was approved by the ethics committee of each investigator's institute or hospital.

Provenance and peer review Not commissioned; externally peer reviewed.

Open Access This is an Open Access article distributed in accordance with the Creative Commons Attribution Non Commercial (CC BY-NC 4.0) license, which permits others to distribute, remix, adapt, build upon this work non-commercially, and license their derivative works on different terms, provided the original work is properly cited and the use is non-commercial. See: http://creativecommons.org/ licenses/by-nc/4.0/

(c) Article author(s) (or their employer(s) unless otherwise stated in the text of the article) 2018. All rights reserved. No commercial use is permitted unless otherwise expressly granted.

\section{REFERENCES}

1 Richette P, Doherty M, Pascual E, et al. 2016 updated EULAR evidence-based recommendations for the management of gout. Ann Rheum Dis 2017;76:29-42.

2 Kuo CF, Grainge MJ, Zhang W, et al. Global epidemiology of gout: prevalence, incidence and risk factors. Nat Rev Rheumatol 2015:11:649-62.

3 Kuo CF, Grainge MJ, Mallen C, et al. Rising burden of gout in the UK but continuing suboptimal management: a nationwide population study. Ann Rheum Dis 2015;74:661-7.

4 Abeles AM. Hyperuricemia, gout, and cardiovascular disease: an update. Curr Rheumatol Rep 2015;17:13

5 Kuo CF, Grainge MJ, Mallen C, et al. Comorbidities in patients with gout prior to and following diagnosis: case-control study. Ann Rheum Dis 2016;75:210-7.

6 Perez-Ruiz F, Becker MA. Inflammation: a possible mechanism for a causative role of hyperuricemia/gout in cardiovascular disease. Curr Med Res Opin 2015;31(Suppl 2):9-14. 
7 Shoji A, Yamanaka H, Kamatani N. A retrospective study of the relationship between serum urate level and recurrent attacks of gouty arthritis: evidence for reduction of recurrent gouty arthritis with antihyperuricemic therapy. Arthritis Rheum 2004;51:321-5.

8 Perez-Ruiz F, Calabozo M, Pijoan Jl, et al. Effect of urate-lowering therapy on the velocity of size reduction of tophi in chronic gout. Arthritis Rheum 2002;47:356-60.

9 Schumacher HR Jr, Becker MA, Lloyd E, et al. Febuxostat in the treatment of gout: 5-yr findings of the FOCUS efficacy and safety study. Rheumatology 2009;48:188-94.

10 Schumacher HR Jr, Becker MA, Wortmann RL, et al. Effects of febuxostat versus allopurinol and placebo in reducing serum urate in subjects with hyperuricemia and gout: a 28-week, phase III, randomized, double-blind, parallel-group trial. Arthritis Rheum 2008;59:1540-8.

11 Sundy JS, Baraf HS, Yood RA, et al. Efficacy and tolerability of pegloticase for the treatment of chronic gout in patients refractory to conventional treatment: two randomized controlled trials. JAMA 2011:306:711-20.

12 Becker MA, Schumacher HR Jr, Wortmann RL, et al. Febuxostat compared with allopurinol in patients with hyperuricemia and gout. N Engl J Med 2005;353:2450-61.

13 Lee S, So MW. Adherence with urate-lowering therapies among male patients with gout in a routine clinical setting. Mod Rheumatol 2016;26:950-5.

14 McGowan B, Bennett K, Silke C, et al. Adherence and persistence to urate-lowering therapies in the Irish setting. Clin Rheumatol 2016;35:715-21.

15 Becker MA, MacDonald PA, Hunt BJ, et al. Determinants of the clinical outcomes of gout during the first year of urate-lowering therapy. Nucleosides Nucleotides Nucleic Acids 2008;27:585-91.

16 Briesacher BA, Andrade SE, Fouayzi $\mathrm{H}$, et al. Comparison of drug adherence rates among patients with seven different medical conditions. Pharmacotherapy 2008:28:437-43.

17 Riedel AA, Nelson M, Joseph-Ridge N, et al. Compliance with allopurinol therapy among managed care enrollees with gout: a retrospective analysis of administrative claims. J Rheumatol 2004;31:1575-81.

18 Zandman-Goddard G, Amital H, Shamrayevsky N, et al. Rates of adherence and persistence with allopurinol therapy among gout patients in Israel. Rheumatology 2013;52:1126-31.

19 Seth R, Kydd AS, Falzon L, et al. Preventing attacks of acute gout when introducing urate-lowering therapy: a systematic literature review. J Rheumato/ Supp/ 2014;92:42-7.

20 Khanna D, Khanna PP, Fitzgerald JD, et al. 2012 American College of Rheumatology guidelines for management of gout. Part 2: therapy and antiinflammatory prophylaxis of acute gouty arthritis. Arthritis Care Res 2012;64:1447-61.

21 van Echteld I, Wechalekar MD, Schlesinger N, et al. Colchicine for acute gout. Cochrane Database Syst Rev 2014;8:CD006190.

22 Terkeltaub RA, Furst DE, Bennett K, et al. High versus low dosing of oral colchicine for early acute gout flare: twenty-four-hour outcome of the first multicenter, randomized, double-blind, placebo-controlled, parallel-group, dose-comparison colchicine study. Arthritis Rheum 2010:62:1060-8.

23 Finkelstein Y, Aks SE, Hutson JR, et al. Colchicine poisoning: the dark side of an ancient drug. Clin Toxicol 2010;48:407-14.
24 Schlesinger N. Treatment of chronic gouty arthritis: it is not just about urate-lowering therapy. Semin Arthritis Rheum 2012;42:155-65.

25 Kamatani N, Fujimori S, Hada T, et al. Placebo-controlled double-blind dose-response study of the non-purine-selective xanthine oxidase inhibitor febuxostat (TMX-67) in patients with hyperuricemia (including gout patients) in Japan: late phase 2 clinical study. J Clin Rheumatol 2011;17:\$35-43.

26 Kamatani N, Fujimori S, Hada T, et al. An allopurinol-controlled, randomised, doubledummy, double-blind, parallel between-group, comparative study of febuxostat (TMX-67), a non-purine-selective inhibitor of xanthine oxidase, in patients with hyperuricemia including those with gout in Japan: phase 3 clinical study. J Clin Rheumatol 2011;17:S13-S18.

27 Wallace SL, Robinson H, Masi AT, et al. Preliminary criteria for the classification of the acute arthritis of primary gout. Arthritis Rheum 1977;20:895-900.

28 Yamamoto T, Hidaka Y, Inaba $M$, et al. Effects of febuxostat on serum urate level in Japanese hyperuricemia patients. Mod Rheumatol 2015;25:779-83.

29 Pocock SJ. Clinical Trials: A Practical Approach. New York: John Wiley \& Sons, 1983.

30 Kamatani N, Fujimori S, Hada T, et al. Placebo-controlled, double-blind study of the non-purine-selective xanthine oxidase inhibitor febuxostat (TMX-67) in patients with hyperuricemia including those with gout in Japan: phase 3 clinical study. J Clin Rheumatol 2011;17:S19-26.

31 Bender R, Lange S. Adjusting for multiple testing--when and how? J Clin Epidemiol 2001:54:343-9.

32 R Core Team. $R$ : A language and environment for statistical computing. $R$ Foundation for Statistical Computing. Vienna, Austria, 2014. http://www.R-project. org/

33 Komoriya K, Hoshide S, Takeda K, et al. Pharmacokinetics and pharmacodynamics of febuxostat (TMX-67), a non-purine selective inhibitor of xanthine oxidase/xanthine dehydrogenase (NPSIXO) in patients with gout and/or hyperuricemia. Nucleosides Nucleotides Nucleic Acids 2004;23:1119-22.

34 Borstad GC, Bryant LR, Abel MP, et al. Colchicine for prophylaxis of acute flares when initiating allopurinol for chronic gouty arthritis. J Rheumatol 2004;31:2429-32.

35 Khanna D, Fitzgerald JD, Khanna PP, et al. 2012 American College of Rheumatology guidelines for management of gout. Part 1: systematic nonpharmacologic and pharmacologic therapeutic approaches to hyperuricemia. Arthritis Care Res 2012;64:1431-46

36 Perez-Ruiz F. Treating to target: a strategy to cure gout. Rheumatology 2009;48:ii9-ii14

37 Stamp LK. Safety profile of anti-gout agents: an update. Curr Opin Rheumatol 2014;26:162-8.

38 Taylor WJ, Shewchuk R, Saag KG, et al. Toward a valid definition of gout flare: results of consensus exercises using Delphi methodology and cognitive mapping. Arthritis Rheum 2009;61:535-43.

39 Gaffo AL, Schumacher HR, Saag KG, et al. Developing a provisional definition of flare in patients with established gout. Arthritis Rheum 2012;64:1508-17.

40 Saag KG, Whelton A, Becker MA, et al. Impact of febuxostat on renal function in gout subjects with moderate-to-severe renal impairment. Arthritis Rheum 2016:68:2035-43. 